\title{
PERCEPÇÃO DE COMPETÊNCIA COMPARATIVA EM CRIANÇAS PRATICANTES DE ATIVIDADES ESPORTIVAS COLETIVAS
}

\author{
Felipe Queiroz do Vale \\ Daniel Vicentini de Oliveira ${ }^{2}$ \\ José Roberto Andrade do Nascimento Junior ${ }^{3}$ \\ Lenamar Fiorese Vieira ${ }^{4}$ \\ Carla Thamires Laranjeira Granja ${ }^{5}$ \\ Vanildo Rodrigues Pereira ${ }^{6}$
}

\begin{abstract}
VALE, F. Q. do; OLIVEIRA, D. V. de; NASCIMENTO JUNIOR, J. R. A. do; VIEIRA, L. F.; GRANJA, C. T. L.; PEREIRA, V. R. Percepção de competência comparativa em crianças praticantes de atividades esportivas coletivas. Arq. Cienc. Saúde UNIPAR, Umuarama, v. 22, n. 3, p, 165-169, set./dez. 2018.
\end{abstract}

\begin{abstract}
RESUMO: Este estudo teve por objetivo investigar a percepção de competência em crianças praticantes de atividades esportivas coletivas. Participaram da pesquisa 76 crianças com idade entre 10 e 12 anos, de ambos os sexos, praticantes de esportes (Handebol, Basquetebol, Voleibol e Futsal). Como instrumento foi utilizado a Escala de Percepção de Competência de Harter. Os dados foram analisados por meio da estatística descritiva e inferencial, sendo significância adotada de $p<0,05$. Os resultados evidenciaram diferença significativa na competência física $(\mathrm{p}=0,04)$, indicando que os meninos apresentaram maior percepção nesta competência em comparação às meninas. Concluiu-se que, na faixa etária de 10 a 12 anos, os meninos conferem maior importância à aparência física do que as meninas.

PALAVRAS-CHAVE: Atividade motora. Desenvolvimento humano. Psicologia do esporte.
\end{abstract}

\section{PERCEPTION OF COMPARATIVE COMPETENCE IN CHILDREN PRACTICING COLLECTIVE SPORTS ACTIVITIES}

ABSTRACT: This study aimed at investigating the perception of competence in children practicing collective sports activities. A total of 76 children aged between 10 and 12 years, both male and female, practicing sports (Handball, Basketball, Volleyball and Futsal) participated in the study. The study used the Harter Competence Perceptual Scale. Data were analyzed using descriptive and inferential statistics, with significance set at $\mathrm{p}<0.05$. The results showed a significant difference in physical competence $(\mathrm{p}=0.04)$, indicating that boys presented a higher perception in this competence when compared to girls. It can be concluded that, in the 10- to 12-year age group, boys consider physical appearance more important when compared to girls.

KEYWORDS: Human development. Motor activity. Sports psychology.

\section{Introdução}

Competência é uma palavra do senso comum, utilizada para designar uma pessoa qualificada para realizar alguma coisa. O seu oposto, ou o seu antônimo, não implica apenas a negação desta capacidade, mas guarda um sentimento pejorativo, depreciativo (FLEURY; FLEURY, 2001). Tal palavra substantiva indica aquele que detêm faculdade, capacidade, suficiência para realizar algo, ou seja, quem for mais, quem faz melhor, segundo os dicionários on-line Michaelis e Priberam a qual Fleury e Fleury (2001) designam para pessoa qualificada.

A continuidade e persistência em continuar uma atividade depende de vários fatores, entre estes destacam-se a capacidade de ter estímulo e sentir-se motivado para alcançar o êxito na execução de uma tarefa. Nos esportes isso não é diferente. Diversos estudos indicam que o estímulo, a motivação, a percepção de obtenção de êxito faz com que a criança inicie as práticas esportivas e continuem. Dessa forma, a realização das ações esportivas passa pela percepção de competência (VILLWOCK; VALENTINI, 2007).

Nos dias atuais, entender o estímulo que leva as crianças iniciarem e prosseguirem no esporte torna-se uma tarefa de suma importância na identificação dos motivos que os conduzem ao êxito, fracasso ou desistência da modalidade esportiva. A criança motivada tende participar de atividades, realizando-as arduamente e persistindo nas mesmas por um longo período de tempo na busca da competência. Segundo Villwock e Valentini (2007), ao perceber-se competente, a criança aprecia o desafio com satisfação mantendo-se engajada; tornando-se mais competente.

Segundo Valentini (2002), a competência pode ser entendida como o julgamento expresso pelo indivíduo relativo a uma capacidade realizada, e pode ser expressa pela criança na infância em domínios específicos do comportamento humano (cognitivo, social ou motor); sendo, portanto, multidimensional a qual permite verificar em qual domínio a criança investiu, ou está investindo, maior energia e esforço para tornar-se competente. Entretanto, as mesmas podem mudar em decorrência das experiências dependendo de suas conquistas.

Quanto maior o nível de realizações ou conquistas, possivelmente o indivíduo irá perceber-se competente no específico domínio que sua ação foi eficiente. Ao perceber-se competente, apreciando o desafio com satisfação, mantendo-se engajada e tornando-se mais competente, levando o indivíduo a sentir prazer em descobrir o que consegue fazer bem. Desta forma, a criança motivada tende participar de ativi-

DOI: 10.25110 /arqsaude.v22i3.2018.6350

${ }^{1}$ Graduado em Educação Física; Universidade Estadual de Maringá (UEM). felipe_q_v@hotmail.com

${ }^{2}$ Graduado em Educação Física e Fisioterapia; Mestre em Promoção de Saúde; Doutorando em Gerontologia; Faculdade Metropolitana de Maringá (FAM-

MA). Avenida Londrina, 934, apto 1907, torre A. CEP 87050-730. Maringá, Paraná. (44) 99942-8575. d.vicentini@hotmail.com

${ }^{3}$ Graduado em Educação Física; Doutor em Educação Física; Universidade Federal do Vale do São Francisco (UNIVASF). jroberto.jrs01@gmail.com

${ }^{4}$ Graduada em Educação Física; Doutora em Ciência do Movimento Humano; Universidade Estadual de Maringá (UEM). 1fvieira@uem.br

${ }^{5}$ Universidade Federal do Vale do São Francisco (UNIVASF) - thamiresgranja1@gmail.com

${ }^{6}$ Graduado em Educação Física; Doutor em Aprendizagem Motora; Universidade Estadual de Maringá (UEM). vrpereira@uem.br - 
dades, realizando-as arduamente e persistindo nas mesmas por um longo período de tempo na busca da competência (HARTER, 1981).

Outro fator que influencia a percepção de competência é a interação social, ao ponto de que, muitas vezes, as mudanças nas percepções de competência das crianças são associadas às percepções de competência dos amigos. O encorajamento positivo de pais e professores e a comparação com os pares quando livres do julgamento contribuem para a melhora da própria percepção de competência (ALTERMATT; POMERANTZ, 2003). Para Valentini (2002), crianças que demonstram curiosidade, que apreciam as etapas de aprendizagem e de resolução dos desafios com autonomia, também se sentem competentes em suas habilidades e quanto mais competentes se percebem, mais positivas são suas reações e mais motivadas se tornam para a realização de novas tarefas. Como defendido por Vygotsky (1998), a criança utiliza a imitação em situações nas quais interage com determinadas tarefas que não conseguiria realizar por si só, visto que suas funções psicológicas ainda estão em amadurecimento (CHAIKLIN, 2011).

Quando a criança ou adolescente possui dificuldade de desempenhar certas habilidades básicas de jogo e de esportes com eficiência, e experimentam fracassos repetidos, elas podem se sentir completamente derrotadas. Se a tarefa for considerada importante, então a competência na sua execução terá impacto sobre a autoestima. Mais especificamente, a percepção de competência pode ter impacto significativo na competência real e ambas promovem a autoconfiança, e esta por sua vez tem potencial para melhorar aspectos dimensionais do autoconceito e da autoestima do indivíduo (GALLAHUE; OZMUN, 2005).

Para Lopes e Nunomura (2007), compreender os fatores motivacionais que levam crianças à prática esportiva facilita o direcionamento das metas do mesmo com o esporte, além de contribuir, também, para a elaboração das atividades de treinamento e das respectivas estratégias as quais visem evitar o abandono precoce do esporte. Dessa forma, é possível perceber a importância da competência sobre a construção de aspectos fisio e psicológicos, sendo de fundamental importância estudos que explorem o desenvolvimento dessa característica através da inclusão da criança no contexto esportivo.

Diante disto, este estudo teve por objetivo investigar a percepção de competência em crianças praticantes de atividades esportivas coletivas em função do sexo.

\section{Material e Métodos}

Esta pesquisa caracteriza-se como quantitativa do tipo descritiva, observacional e transversal, submetida e aprovada pelo Comitê Permanente de Ética em Pesquisa com Seres Humanos da Universidade Estadual de Maringá (UEM) sob o parecer $n^{\circ} 339 / 2011$, CAAE 0227.0.093.00011 .

A amostra foi composta por 76 crianças de 10 a 12 anos praticantes de diferentes modalidades esportivas coletivas (Handebol, Basquetebol, Voleibol e Futsal). A escolha se deu pelo fato de serem estas as modalidades de maior difusão em idade escolar, junto ao trabalho realizado com maior ênfase pela da disciplina de Educação Física nas escolas públi- cas e privadas da cidade de Maringá no estado do Paraná. A seleção da amostra foi não-probabilística por conveniência. Como critérios para inclusão foram adotados: a criança deveria ter entre 10 e 12 anos; ser praticante de esportes coletivos (handebol, basquetebol, voleibol ou futsal) a pelo menos seis meses. As crianças praticavam estas modalidades fora das aulas de educação física da escola, em clubes, centros esportivos e academias.

Para verificar o nível de percepção de competência das crianças, foi utilizada a Escala de Percepção de Competência de Harter (1985), adaptada e validada para a língua portuguesa por Valentini et al. (2010). Tal instrumento contém 36 itens distribuídos por seis subescalas: cognitiva, afetiva, motora, aparência física, conduta comportamental e global. A escala é apresentada em pranchas contendo duas figuras colocadas uma ao lado da outra; uma figura corresponde a uma criança competente e outra uma criança não tão competente. A criança primeiramente deve selecionar qual figura mais se parece com ela e então decidir se é muito ou pouco parecida com a criança da figura. Os itens foram pontuados seguindo uma escala Likert de 4 pontos, usada em pesquisas de opinião, variando num escore de 1 "baixa competência" e 4 "alta competência". A análise da consistência interna do questionário por meio do alfa de Cronbach foi $\alpha=$ 0,74 , evidenciando forte confiabilidade dos dados.

Inicialmente, as escolas foram contatadas para assinarem o termo de autorização para realização do estudo. Foram encaminhados os Termos de Consentimento Livre e Esclarecido para os pais responsáveis, e mediante aceite, foram recolhidos contendo a data, o local e o horário para esclarecimentos do estudo e preenchimento dos questionários. Após este procedimento a Escala de Percepção de Competência Física foi aplicada nas crianças no local de treino da escola, cedido pela coordenação. As crianças foram avaliadas coletivamente após, conhecer as formas de preenchimento do questionário.

Para análise dos dados, utilizou-se a estatística descritiva (medianas e intervalo interquartílico) por meio dos parâmetros estatísticos de acordo com a normalidade dos dados demonstrada pelo teste Kolmogorov-smirnov. Diante da não normalidade entre os dados, o teste "U" de Mann-Whitney foi utilizado para comparar a percepção de competência entre o sexo biológico: meninos e meninas, enquanto para as correlações entre percepção de competência foi utilizado o coeficiente de correlação de Spearman (r) e a consistência interna instrumental a base alfa de Cronbach. Teste de significância adotado foi de $\mathrm{p}<0,05$.

\section{Resultados}

Constatamos que meninos evidenciaram maior percepção na competência física $(p=0,04)$ apresentando $\mathrm{Md}=3,10$ enquanto as meninas obtiveram $\mathrm{Md}=2,70$. As competências cognitiva, afetiva, motora, comportamental e valor global não apresentaram diferenças significativas de acordo com dados tabela 1. 
Tabela 1: Comparação da percepção de competência em função do sexo.

\begin{tabular}{|c|c|c|c|c|c|}
\hline \multirow[t]{2}{*}{ Dimensões } & \multicolumn{2}{|c|}{ MASCULINO } & \multicolumn{2}{|c|}{ FEMININO } & \multirow[t]{2}{*}{$P$} \\
\hline & Md & Q1-Q3 & Md & Q1-Q3 & \\
\hline Cognitiva & 2,60 & $2,35-3,07$ & 2,80 & $2,60-3,10$ & 0,39 \\
\hline Afetiva & 2,60 & $2,52-2,80$ & 2,70 & $2,60-3,10$ & 0,31 \\
\hline Motora & 2,80 & $2,60-3,25$ & 3,00 & $2,60-3,25$ & 0,62 \\
\hline Física & 3,10 & $2,80-3,60$ & 2,70 & $2,15-3,45$ & $0,04 *$ \\
\hline $\begin{array}{l}\text { Comporta- } \\
\text { mental }\end{array}$ & 2,60 & $2,30-3,00$ & 2,70 & $2,60-3,10$ & 0,25 \\
\hline $\begin{array}{l}\text { Valor } \\
\text { Global }\end{array}$ & 3,40 & $3,00-3,75$ & 3,30 & $2,60-3,25$ & 0,86 \\
\hline
\end{tabular}

Para verificar as relações entre os domínios de percepção de competência, optou-se por fazer tal análise estratificada por sexo com o intuito de se verificar se as relações são diferentes para meninos e meninas. Ao analisar a correlação entre as competências dos meninos (Tabela 2), verificou-se correlação significativa $(\mathrm{p}<0,05)$, positiva e moderada $(\mathrm{r}>$ $0,40)$ entre as seguintes competências: Física e Global $(\mathrm{r}=$ $0,65)$, Afetiva e Global $(\mathrm{r}=0,56)$. Também foi encontrada correlação significativa $(\mathrm{p}<0,05)$ e fraca $(\mathrm{r}<0,40)$ entre as seguintes competências: Cognitiva e Comportamental $(\mathrm{r}$ $=0,39)$, Afetiva e Física $(r=0,35)$, Física e Comportamental $(\mathrm{r}=0,33)$.

Tabela 2: Correlação entre as subescalas da percepção de competência dos meninos.

\begin{tabular}{l|c|c|c|c|c|c}
\hline \multicolumn{1}{c|}{ Variáveis } & $\mathbf{1}$ & $\mathbf{2}$ & $\mathbf{3}$ & $\mathbf{4}$ & $\mathbf{5}$ & $\mathbf{6}$ \\
\hline 1. Cognitiva & - & 0,31 & 0,27 & 0,04 & $0,39^{*}$ & 0,25 \\
\hline 2. Afetiva & & - & 0,28 & $0,35^{*}$ & 0,24 & $0,56^{*}$ \\
\hline 3. Motora & & & - & 0,16 & 0,09 & 0,21 \\
\hline $\begin{array}{l}\text { 4. Física } \\
\text { 5. Comporta- } \\
\text { mental }\end{array}$ & & & & - & $0,33^{*}$ & $0,65^{*}$ \\
\hline $\begin{array}{l}\text { 6. Valor } \\
\text { Global }\end{array}$ & & & & - & 0,29 \\
\hline * Correlacão significativa & & & & & - \\
\hline
\end{tabular}

* Correlação significativa com valores $\mathrm{p}<0,05$ (nível de significância).

Ao analisar a correlação entre as competências das meninas (Tabela 3 ), verificou-se correlação significativa ( $p$ $<0,05)$, positiva e moderada $(\mathrm{r}>0,40)$ entre as seguintes competências: Cognitiva e Comportamental $(\mathrm{r}=0,66)$, Física e Comportamental $(\mathrm{r}=0,54)$, Física e Global $(\mathrm{r}=0,50)$, Comportamental e Global $(\mathrm{r}=0,46)$.
Tabela 3: Correlação entre as subescalas da percepção de competência das meninas.

\begin{tabular}{l|c|c|c|c|c|c}
\hline \multicolumn{1}{c|}{ Variáveis } & $\mathbf{1}$ & $\mathbf{2}$ & $\mathbf{3}$ & $\mathbf{4}$ & $\mathbf{5}$ & $\mathbf{6}$ \\
\hline 1. Cognitiva & - & $0,36^{*}$ & 0,23 & 0,32 & $0,66^{*}$ & 0,27 \\
\hline 2. Afetiva & & - & 0,22 & 0,25 & 0,28 & 0,23 \\
\hline 3. Motora & & & - & 0,30 & 0,30 & 0,16 \\
\hline $\begin{array}{l}\text { 4. Física } \\
\text { 5. Comporta- } \\
\text { mental }\end{array}$ & & & & - & $0,54^{*}$ & $0,50^{*}$ \\
\hline $\begin{array}{l}\text { 6. Valor } \\
\text { Global }\end{array}$ & & & & & - & $0,46^{*}$ \\
\hline
\end{tabular}

* Correlação significativa com valores $\mathrm{p}<0,05$.

Também foi encontrada correlação significativa ( $p$ $<0,05)$ e fraca $(\mathrm{r}<0,40)$ entre as competências Cognitiva e Afetiva $(\mathrm{r}=0,36)$.

\section{Discussão}

Nos estudos de Weiss e Amorose (2005), afirma-se que a importância de domínios específicos na autopercepção é o ponto alto para algumas teorias as quais identificam a competência percebida como um determinante significante de realização relacionada a comportamentos, pensamentos e respostas afetivas. Assim funciona a percepção de competência, ou seja, as crianças que alcançam maiores níveis de competência, tendem a projetar-se com mais determinação perante atividades em domínios específicos.

Os resultados do presente estudo demonstraram que os meninos apresentaram valores significativamente mais altos na subescala competência física, indicando que estão melhores adaptados em seus atributos físico-corporal do que as meninas. Tal resultado pode ser explicado por resultados encontrados por Silva et al. (2012), que demonstraram diferença entre os minutos anuais de exercício físico que os meninos praticavam em relação às meninas. Resultados similares também foram encontrados no estudo de Grisa (2008).

Resultados dos estudos de Villwock (2005) apontam que o sexo masculino apresenta valores significativamente mais altos na subescala motora/física, e defende que essa diferença apresentada entre os sexos pode estar relacionada à experiência motora vivenciada pelos mesmos ao longo de suas vidas, ou seja, a maior exposição dos meninos às atividades motoras amplas, relacionando o fato a uma questão cultural e histórica de vivência motora.

Segundo Faria (2005), o domínio físico e, muito particularmente, o da aparência física, é um dos domínios que mais contribui para a definição do autoconceito da criança e do adolescente e é fundamental também para a sua autoestima global, ou seja, o domínio físico tem um papel importante, desde a infância, no ajustamento psicossocial do indivíduo, pois as crianças recebem desde muito cedo feedback direto e indireto acerca da sua atratividade física, da sua condição física, do seu peso, da sua altura e da forma como se vestem ou se apresentam. Nascimento (1999) defende que a percepção de competência na infância parece ter base essencialmente na aceitação pelos pares. Dessa forma, se sentir competente fisicamente acarreta na infância acarreta uma aceitação no ambiente em que estão inseridos, influenciando 
assim, a competência global das crianças.

No entanto, os outros domínios não apresentaram diferenças significativas, demonstrando níveis satisfatórios de percepção de competência e confirmando o propósito de que atualmente meninos e meninas não estão mais se diferenciando na forma de se autojulgar. Os resultados encontrados vão ao encontro a outras pesquisas realizadas (VALENTINI, 2002; ALMEIDA et al., 2009), que encontram competência moderada, sem diferenças entre os sexos. Valentini (2002) sugere que quando são oferecidas oportunidades para o desenvolvimento adequado das capacidades, meninos e meninas apresentarão percepções de competência similares. Tais resultados corroboram com o estudo de Valentini (2009), que demonstrou habilidades de locomoção e desempenho motor similar entre meninos e meninas.

Em relação ao valor global avaliado no estudo, não obtivemos diferenças significativas, no entanto, obtivemos os maiores valores, indicando alto nível de competência, o que demonstra que ambos estão confiantes no momento com o seu jeito de ser. A criança, ao participar de uma tarefa ou meta, seja ela de qualquer origem, afetiva, cognitiva, física ou motora, se depara com sentimentos que muitas vezes não são conscientes. No entanto, dependem de experiências prévias para o seu autojulgamento. A percepção de competência é um veículo que impulsiona a prática ou não de atividades, tais como a atividade esportiva. Um estudo de Vieira (1993) demonstra que atletas com alta percepção de competência atlética têm como principal motivo para a prática do esporte o desenvolvimento de suas habilidades, e também que os atletas com uma percepção de aparência física elevada citaram os motivos de aptidão como principais para a prática.

Ressalta-se a importância da competência afetiva para os meninos e da comportamental para as meninas. Ou seja, para as meninas, o sucesso na aceitação de regras de comportamento social tem relação expressiva com a percepção de competências global, física e cognitiva. Já para os meninos, a sensação de capacidade de estabelecer vínculos e parcerias se relaciona expressivamente na competência física e global.

Tais achados apontam que principalmente os aspectos físicos, tanto para os meninos quanto para as meninas, são os fatores mais relacionados com as demais competências, enfatizando a importância de se desenvolver as diferentes capacidades físicas durante a infância. Villwock (2007) afirma que o indivíduo que se percebe competente para realizar uma determinada atividade motiva-se a permanecer na mesma, se sua percepção de competência for semelhante à sua competência real. O que determina a sua influência sobre o indivíduo são as atividades vivenciadas previamente, a interação social e o tipo de instrução proporcionada aos iniciantes durante o processo de desenvolvimento.

Desta forma, Grisa (2008) afirma que, a percepção de competência se desenvolve a partir de diversas fontes, sendo elas o feedback dos professores, de suporte oferecido pelos pais e, por último, da influência de colegas enquanto agentes socializadores. Os pais atuam como transmissores de informações acerca do valor que eles postulam sobre as ações, enfatizando ou restringindo a participação de seu filho. Os professores influenciam a partir do contexto oferecido ao aluno, juntamente com o clima motivacional, o feedback, as instruções, as críticas e os elogios. Os amigos/ colegas influenciam no sentido da aceitação social da relação amistosa estabelecida a partir da popularidade adquirida pelo envolvimento bem-sucedido na atividade. Assim, todo o contexto em que a criança está inserida tem influência na sua percepção de competência, sendo de fundamental importância o papel desses agentes sociais.

\section{Conclusão}

Por meio dos resultados encontrados, pode-se concluir que na faixa etária de 10 a 12 anos, os meninos conferem maior importância à aparência física do que as meninas. Ressalta-se que para as meninas, o sucesso na aceitação de regras de comportamento social demonstra foi um fator de grande relevância, enquanto para os meninos, a sensação de capacidade de estabelecer vínculos e parcerias demonstrou ser o mais expressivo.

Como implicações práticas, acredita-se que este estudo possa alavancar novas formas de investigar a percepção de competência entre meninos e meninas dentro das modalidades esportivas coletivas. Para tanto, salienta-se a necessidade de pesquisas mais profundas nesse método, em populações maiores, e com maiores afinidades culturais e sociais para que possamos identificar os motivos que podem interromper a prática esportiva de crianças e jovens praticantes.

\section{Referências}

ALMEIDA, G.; VALENTINI, C. F.; BERLEZE, A. Percepções de Competência: Um Estudo com Crianças e Adolescentes do Ensino Fundamental. Revista Movimento, v. 15, n. 1, p. 71-97, 2009.

\section{ALTERMATT, E. R.; POMERANTZ, E. M. The}

development of competence-related and motivational beliefs: an investigation of similarity and influence among friends. Journal of Educational Psychology, v. 95, n.1. 2003.

BRAUNER, L. M.; VALENTINI, N. C. Análise do desempenho motor de crianças participantes de um programa de atividades físicas. Journal of Phisical Education, v. 20, n. 2, p. 205-216, 2009.

ARENA, S. S. BÖHME, M. T. S. Programas de iniciação e especialização esportiva na grande São Paulo. Revista Paulista de Educação Física, v. 14, n. 2, p. 184-195, 2000.

BENETTI, G.; SCHNEIDER, P.; MEYER, F.; Os benefícios do esporte e a importância da treinabilidade da força muscular de pré- puberes atletas de voleibol. Revista brasileira de cineantropometria e desempenho humano, v. 7, n. 2, p. 87-93, 2003.

CHAIKLIN, S. A zona de desenvolvimento próximo na análise de Vigotski sobre aprendizagem e ensino. Psicologia em estudo, v. 16, n. 4, p. 659-675, 2011.

FARIA, L. Desenvolvimento do autoconceito físico nas crianças e nos adolescentes. Análise Psicológica, v. 23, n. 4, p. 361-371, 2005. 
FIORESE, L. V. A relação entre a percepção de competência

esportiva. Revista de Educação Física/UEM, v. 4, n. 1, p 40-44, 1993.

FLEURY, M. T. L.; FLEURY, A. Construindo o conceito de competência. Revista de Administração Contemporânea, v. 5, edição especial, p. 183-1996, 2001.

GAllahUE, D. L.; OZMUN, J. C. Compreendendo o Desenvolvimento Motor: bebês, crianças, adolescentes e adultos. São Paulo: Phorte, 2005.

GRAY, H. M. et al. P300 as an index of attention to self-relevant stimuli. Journal of Experimental Social Psychology, v. 40, n. 2, p. 216-224, 2003.

HARTER, S. The developmental of competence motivacion in the mastery ofcognitive and physical skills: Is there still a place for joy. Psychology of motorbehavior and sport, 1980.

MACHADO, D. R. L.; BARBANTI, V. J. Maturação esquelética e crescimento em crianças e adolescentes. Revista Brasileira de Cineantropometria e Desempenho Humano, v. 9, n. 1, p. 1220, 2007.

RÉ, N. A. Características do futebol e do futsal: implicações para o treinamento de adolescentes e adultos jovens. Revista Digital. Año 13 - No 127. Buenos Aires, 2008.

SILVA, R. B. et al. Relação da prática de exercícios físicos e fatores associados às regulações motivacionais de adolescentes brasileiros. Motricidade, v. 8, n. 2, p. 8-21, 2012.

VALENTINI, N. C. A influência de uma intervenção motora no desempenho motor e na percepção de competência de crianças com atrasos motores. Revista Paulista de Educação Física. São Paulo. v. 16, n. 1, p. 61-7, 2002.

VALENTINI, N. C. et al. Validação brasileira da escala de autopercepção de Harter para crianças. Psicologia: Reflexão e Crítica, v. 23, n. 3, p. 411-419, 2010.

VILLWOCK, G.; VALENTINI, N. C. Percepção de competência atlética, orientação motivacional e competência motora em crianças de escolas públicas: estudo desenvolvimentista e correlacional. Revista Brasileira de Educação Física e Esporte, v. 21, n. 4, p. 245-257, 2007.

VYGOTSKY, L. S. (1998b). The problem of age (M. Hall, Trans.). In R. W. Rieber (Ed.), The collected works of L. S. Vygotsky: (Vol. 5. Child psychology) (pp. 187-205). New York: Plenum Press. (Original publicado em 1933-1934).

WEISS, M. R.; AMOROSE, A. J. Children's SelfPerceptions in the Physical Domain: Between- and Within-Age Variability in Level, Accuracy, and Sources of Perceived Competence. Journal of Sport \& Exercise Psychology. v. 27, p. 226-244, 2005. 